\title{
A Three-Phase State Estimation in Unbalanced Distribution Networks with Switch Modelling
}

\author{
Ankur Majumdar \\ Student Member, IEEE \\ Dept of Electrical and Electronic Engineering \\ Imperial College London \\ London, U.K. \\ ankur.majumdar@imperial.ac.uk
}

\author{
Bikash C. Pal \\ Fellow, IEEE \\ Dept of Electrical and Electronic Engineering \\ Imperial College London \\ London, U.K. \\ b.pal@imperial.ac.uk
}

\begin{abstract}
State estimation has become an important task in modern energy/ distribution management systems. However, the state estimation is not very popular in modern unbalanced threephase distribution systems. This paper proposes a method for three-phase state estimation with detailed three-phase modelling of system components including switches and star and delta connected loads. This method is then tested on a standard IEEE 13-bus system and the results are compared with load flow results.
\end{abstract}

Keywords—state estimation, distribution management systems (DMS), three-phase modelling, weighted least squares (WLS), switch modelling

\section{INTRODUCTION}

With the influx of phasor measurement units (PMUs), intelligent metering etc. in transmission systems and smart meters with information and communication technology (ICT) infrastructure in distribution systems, power systems now-adays need to be monitored and controlled efficiently [1]. To enable this, the states of the system need to be observed properly. This would help to influence the operational decisions and thus, to avoid contingency and cascaded tripping. It is done through an energy/ distribution management system (EMS/DMS) function- the state estimation (SE) [2], [3]. This function estimates the bus voltages and angles based on the available measurements, network data and topology information. Figure I shows a typical DMS architecture.

In transmission systems, the state estimation concept is well established but in distribution systems due to the absence of sufficient measurements and unbalanced and asymmetric nature of the system, it was not mandatory to have a state estimation function. But with growing number of controllable devices and the incorporation of smart meters in the system, state estimation is becoming important in distribution network operation.

As a starting point, the solution methodology mainly focuses on weighted least squares (WLS) estimation technique [4]. But the majority of distribution systems operate under varying degrees of unbalance. Moreover, unlike the transmission system, the distribution system is radial in nature and has a higher $R / X$ ratio. Therefore, the fast decoupled method causes numerical instability when applied to distribution systems [5]. Hence, this has paved the way for the need of unbalanced three-phase state estimation rather than singlephase state estimation.

Over the years, there have been research on three-phase load flow in distribution systems [6]. However, most of the operational i.e., control and contingency, decisions based on state estimation have been applied to distribution system considering to be balanced [7]. Moreover, the loads considered are constant power and Y-connected loads and the different status (closed/open) of the switches are not considered [8], [9].

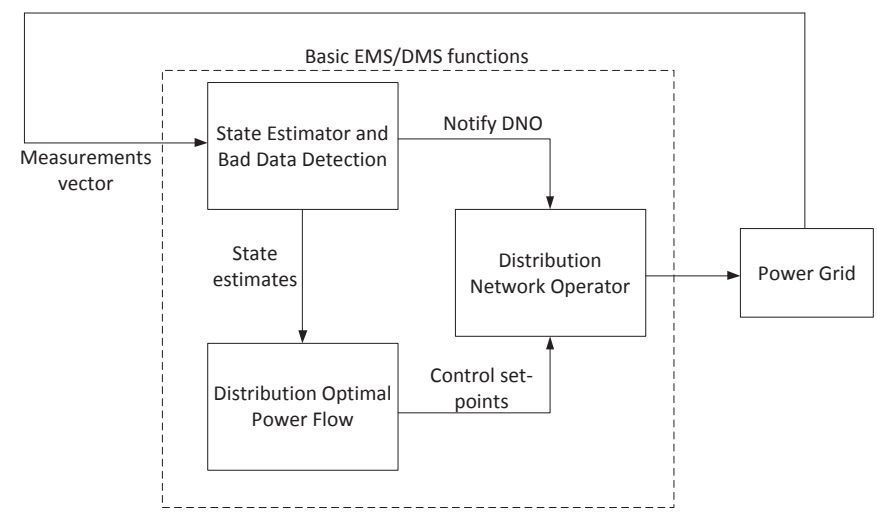

Figure 1. A typical distribution management system architecture

To achieve the accurate estimates of the state variables, this paper presents a weighted least squares based estimator with the detailed modelling of the system components and different types of loads and also considering the different operational status of the switches. The paper is organized as follows. Section II describes the formulation of the problem. Section III discusses the modelling of different components of the distribution system and Section IV demonstrates the results and discussions of the technique applied to 13-bus system. Section V concludes the paper.

\section{Problem Formulation}

The state estimation is a process which estimates realtime states of the system (voltage magnitudes and angles). 
The problem can be looked at as a constrained non-linear optimization problem with the following objective function

$$
J=\left[z_{\text {meas }}-h_{\text {func }}(x)\right]^{T} R^{-1}\left[z_{\text {meas }}-h_{\text {func }}(x)\right]
$$

Subject to:

$$
\begin{array}{r}
c_{e q}(x)=0 \\
c_{\text {ineq }}(x) \leq 0
\end{array}
$$

Where,

$x \quad$ State variables such as voltage magnitudes and angles.

$m \quad$ Number of measurements per phase.

$R \quad$ Measurement error covariance matrix,

$z_{\text {meas }}=\left[\begin{array}{lllllllllll}z_{1}^{a} & z_{1}^{b} & z_{1}^{c} & \ldots & z_{i}^{a} & z_{i}^{b} & z_{i}^{c} & \ldots & z_{m}^{a} & z_{m}^{b} & z_{m}^{c}\end{array}\right]$.

$z_{i} \quad$ Measured value of $i^{\text {th }}$ measurement.

$h_{\text {func }}(x)$ vector of measurement as a function of state $x$

$c_{e q}(x) \quad$ vector of zero injection measurements.

$c_{\text {ineq }}(x) \quad$ vector of inequality constraints.

In three phase system

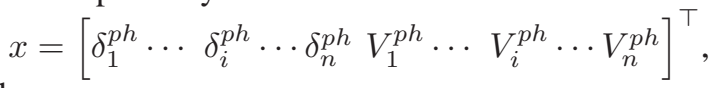

where,

$$
V_{i}^{p h}=\left[\begin{array}{c}
V_{i}^{a} \\
V_{i}^{b} \\
V_{i}^{c}
\end{array}\right], \delta_{i}^{p h}=\left[\begin{array}{c}
\delta_{i}^{a} \\
\delta_{i}^{b} \\
\delta_{i}^{c}
\end{array}\right]
$$

are the three-phase voltage magnitude and voltage angle at bus $i$ respectively.

The measurements are usually considered to be with random errors due to biases, drifts or wrong connections of the measurement devices, i.e. meters. It is assumed that the measurement errors are identically and independently distributed. Hence, the covariance matrix of the errors are given by $R=\operatorname{Cov}(\mathbf{e})=E\left(\mathbf{e e}^{\mathbf{T}}\right)=\operatorname{diag}\left(\sigma_{1}^{2}, \ldots \sigma_{i}^{2}, \ldots, \sigma_{m}^{2}\right)$, where $\sigma_{i}=$ $\left[\begin{array}{lll}\sigma_{i}^{a} & \sigma_{i}^{b} & \sigma_{i}^{c}\end{array}\right]^{\top}$ and $\sigma_{i}^{2}$ is the variance of the $i^{t h}$ measurement error.

In three-phase system, the real power injection $P_{i}^{p h}$ and reactive power injection $Q_{i}^{p h}$ equations at bus $i$ for phase $p h$ can be written as:

$$
\begin{aligned}
P_{i}^{p h}= & V_{i}^{p h} \sum_{l=1}^{3} \sum_{j=1}^{n} V_{j}^{l}\left[G_{i j}^{p h, l} \cos \left(\delta_{i}^{p h}-\delta_{j}^{l}\right)\right. \\
& \left.+B_{i j}^{p h, l} \sin \left(\delta_{i}^{p h}-\delta_{j}^{l}\right)\right] \\
Q_{i}^{p h}= & V_{i}^{p h} \sum_{l=1}^{3} \sum_{j=1}^{n} V_{j}^{l}\left[G_{i j}^{p h, l} \sin \left(\delta_{i}^{p h}-\delta_{j}^{l}\right)\right. \\
& \left.-B_{i j}^{p h, l} \cos \left(\delta_{i}^{p h}-\delta_{j}^{l}\right)\right]
\end{aligned}
$$

Where $G+j B$ is the system admittance matrix, $n$ is number of buses and $l$ is the number of phases that can be 1,2 or 3 phase. The branch real power flow $P_{i j}^{p h}$ and reactive power flow $Q_{i j}^{p h}$ equations from bus $i$ to bus $j$ for phase $p h$ can be written as follows:

$$
\begin{aligned}
& P_{i j}^{p h}= \\
& V_{i}^{p h} \sum_{l=1}^{3} V_{i}^{l}\left[G_{i j}^{p h, l} \cos \left(\delta_{i}^{p h}-\delta_{i}^{l}\right)+B_{i j}^{p h, l} \sin \left(\delta_{i}^{p h}-\delta_{i}^{l}\right)\right] \\
& -V_{i}^{p h} \sum_{l=1}^{3} V_{j}^{l}\left[G_{i j}^{p h, l} \cos \left(\delta_{i}^{p h}-\delta_{j}^{l}\right)+B_{i j}^{p h, l} \sin \left(\delta_{i}^{p h}-\delta_{j}^{l}\right)\right]
\end{aligned}
$$

$$
\begin{aligned}
& Q_{i j}^{p h}= \\
& -V_{i}^{p h} \sum_{l=1}^{3} V_{i}^{l}\left[G_{i j}^{p h, l} \sin \left(\delta_{i}^{p h}-\delta_{i}^{l}\right)-B_{i j}^{p h, l} \cos \left(\delta_{i}^{p h}-\delta_{i}^{l}\right)\right] \\
& -V_{i}^{p h} \sum_{l=1}^{3} V_{j}^{l}\left[G_{i j}^{p h, l} \sin \left(\delta_{i}^{p h}-\delta_{j}^{l}\right)-B_{i j}^{p h, l} \cos \left(\delta_{i}^{p h}-\delta_{j}^{l}\right)\right]
\end{aligned}
$$

Where,

$V_{i}^{l} \quad$ Voltage magnitude of phase $l$ at bus $i$.

$\delta_{i}^{l} \quad$ Angle of phase $l$ in bus $i$.

\section{A. Equality constraints $c_{e q}(x)$}

The equality constraints are the set of equations corresponding to virtual measurements.

$$
\begin{aligned}
& 0=P_{G i}^{p h}-P_{D i}^{p h}-P_{i}^{p h} \\
& 0=Q_{G i}^{p h}-Q_{D i}^{p h}-Q_{i}^{p h}
\end{aligned}
$$

Where $P_{G i}^{p h}$ and $Q_{G i}^{p h}$ are the real and reactive power injected at bus $i$ respectively, the load demand at the same bus is represented by $P_{D i}^{p h}$ and $Q_{D i}^{p h}$. Indices $n$ is number of buses and $l$ is the number of phases which can be 1,2 or 3 phase.

\section{B. Inequality constraints $c_{\text {ineq }}(x)$}

These are the set of constraints of state variables that represent the system operational and security limits, such as setting upper and lower limits for control variables. The constraints are as follows:

- Bus voltage - Voltage magnitudes at each bus in the network:

$$
V_{\text {min }, i}^{p h} \leq V_{i}^{p h} \leq V_{\max , i}^{p h}
$$

- Bus angle - The bus angle at each bus in the network:

$$
-\delta_{\min , i}^{p h} \leq \delta_{i}^{p h} \leq \delta_{\max , i}^{p h}
$$

Eq. (1) and (2) can be solved by Newton's method, which translates into solving the following equation at each iteration

$$
\left[G\left(x^{k}\right)\right] \Delta x^{k+1}=H^{T} R^{-1}\left[z-h\left(x^{k}\right)\right]
$$

where, $n$ is the total number of buses in the system.

$\Delta x^{k+1}=x^{k+1}-x^{k}$ and $H\left(x^{k}\right)=\left[\frac{\partial h}{\partial x}\right]$ is the Jacobian matrix and $G\left(x^{k}\right)=H^{T}\left(x^{k}\right) R^{-1} H\left(x^{k}\right)$ is the Gain matrix in the $k^{\text {th }}$ iteration. 


\section{THREE-PHASE DISTRIBUTION SYSTEM MODELLING}

The distribution system consists of unsymmetrical network components and unbalanced load. Hence, the single line representation for an unbalanced distribution system does not work. Therefore, the exact three-phase modelling of the network components is necessary.

The following subsections describe the three phase modelling of various components of the network such as line, transformers and switches.

\section{A. Line Modelling}

The distribution system consists of untransposed overhead lines and underground cables which can be three-phase or single and/or two-phase laterals. This combined with the unbalanced loads (single, two or three-phase loads) contribute to the unbalanced nature of the system. Due to the untransposed nature of the lines, it is essential to compute the impedance of the lines accurately. A modified Carson's equation is applied to compute the self and mutual impedance of the lines [10].

$$
\begin{aligned}
& Z_{i i}=r_{i}+0.095+j 0.121 \times\left(\operatorname{Ln} \frac{1}{G M R_{i}}+7.934\right) \Omega / \text { mile } \\
& Z_{i j}=0.095+j 0.121 \times\left(\operatorname{Ln} \frac{1}{D_{i j}}+7.934\right) \Omega / \text { mile }
\end{aligned}
$$

Where,

$$
\begin{array}{ll}
Z_{i i} & \text { Self-impedance of conductor } i \text { in } \Omega / \text { mile. } \\
Z_{i j} & \text { Mutual impedance between conductors } i \text { and } j \text { in } \\
& \Omega / \text { mile. } \\
r_{i} & \text { Resistance of conductor } i \text { in } \Omega / \text { mile. } \\
G M R_{i} & \text { Geometric mean radius of conductor } i \text { in feet. } \\
D_{i j} & \text { Distance between conductors } i \text { and } j \text { in feet. }
\end{array}
$$

The modified Carson's equation also takes into the ground return path (neutral conductor) for the unbalanced currents.

The modified Carson's equations (10) and (11) for a three phase overhead or underground circuit which consists of neut neutral conductors makes the resulting impedance matrix $(3+$ neut $) \times(3+$ neut $)$. However, for most applications, it is necessary to have the $3 \times 3$ phase impedance matrix. Therefore, $(3+n e u t) \times(3+n e u t)$ impedance matrix is broken down to $3 \times 3$ matrix by Kron's reduction as given in (12). In this approach, all the lines will be modelled by $3 \times 3$ phase impedance matrix and for two phase and single phase lines the missing phases are modelled by setting the impedance element to zero.

$$
Z_{i j}=Z_{i j}-\frac{Z_{i n} \times Z_{n j}}{Z_{n n}}
$$

Therefore, for each line between two nodes, there will be a $3 \times 3$ matrix instead of a single element for a single phase balanced system. Hence, the resultant $Y$-bus matrix of the system will be of $(n \times 3) \times(n \times 3)$. The structure of the
$Y$-bus matrix is shown in (13).

$$
Y=\left[\begin{array}{ccccccc}
Y_{11}^{a a} & Y_{11}^{a b} & Y_{11}^{a c} & \ldots & Y_{1 n}^{a a} & Y_{1 n}^{a b} & Y_{1 n}^{a c} \\
Y_{11}^{b a} & Y_{11}^{b b} & Y_{11}^{b c} & \ldots & Y_{1 n}^{b a} & Y_{1 n}^{b b} & Y_{1 n}^{b c} \\
Y_{11}^{c a} & Y_{11}^{c b} & Y_{11}^{c c} & \ldots & Y_{1 n}^{c a} & Y_{1 n}^{c b} & Y_{1 n}^{c c} \\
\vdots & \vdots & \vdots & \ddots & \vdots & \vdots & \vdots \\
Y_{n 1}^{a a} & Y_{n 1}^{a b} & Y_{n 1}^{a c} & \ldots & Y_{n n}^{a a} & Y_{n n}^{a b} & Y_{n n}^{a c} \\
Y_{n 1}^{b a} & Y_{n 1}^{b b} & Y_{n 1}^{b c} & \ldots & Y_{n n}^{b a} & Y_{n n}^{b b} & Y_{n n}^{b c} \\
Y_{n 1}^{c a} & Y_{n 1}^{c b} & Y_{n 1}^{c c} & \ldots & Y_{n n}^{c a} & Y_{n n}^{c b} & Y_{n n}^{c c}
\end{array}\right]
$$

\section{B. Transformer Modelling}

The distribution system generally consists of feeder and distribution transformers which provide the final voltage transformation to the loads. The three phase transformers are modeled by admittance matrix which depends on the connection type. A transformer can be Y-Y, Y- $\Delta, \Delta-\Delta$ etc. In the analysis of the distribution feeder, it is required to model the various three phase transformer connections correctly. The comprehensive calculations of three phase transformers and their various connections can be found in reference [10], [11]. While forming the $Y$-bus, a transformer can be considered as one element between two nodes of the system. Therefore, the transformer contributes to $6 \times 6$ block in the $Y$-matrix.

\section{Switch Modelling}

Switches are considered as branches with zero impedance. It is assumed that the status of the switches, i.e. closed or open, are known beforehand. The operational constraints for the switches are considered as equality constraints as given by $c_{e q}=0$ in equation (2) of the original problem formulation.

- When the switch between bus $i$ and bus $j$ is assumed closed for branch $i$ - $j$, the voltages and angles for bus $i$ and bus $j$ and phase $p h$ for all the three phases are equal.

$$
\begin{gathered}
V_{i}^{p h}-V_{j}^{p h}=0 \\
\delta_{i}^{p h}-\delta_{j}^{p h}=0
\end{gathered}
$$

- When the switch is assumed open between bus $i$ and bus $j$, the active and reactive power flow to the switch will be zero.

$$
\begin{aligned}
& P_{i j}^{p h}=0 \\
& Q_{i j}^{p h}=0
\end{aligned}
$$

\section{Load Modelling}

The loads in distribution systems are generally unbalanced. The loads are three-phase, two-phase or single-phase. They can be connected in grounded Y or ungrounded $\Delta$ configuration. from the point of view of electricity usage, loads can be broadly classified as constant power, constant impedance or constant current loads. They are commonly represented as power consumed per phase and considered to be L-N for Yloads and L-L for $\Delta$-loads. The typical ZIP models for wye and $\Delta$ loads are shown in (16) and (18). 


$$
\begin{gathered}
P_{L}^{p h}=P_{n}^{p h}\left[c_{1}^{P}+c_{2}^{P}\left(\frac{V^{p h}}{V_{n}}\right)+c_{3}^{P}\left(\frac{V^{p h}}{V_{n}}\right)^{2}\right] \\
Q_{L}^{p h}=Q_{n}^{p h}\left[c_{1}^{Q}+c_{2}^{Q}\left(\frac{V^{p h}}{V_{n}}\right)+c_{3}^{Q}\left(\frac{V^{p h}}{V_{n}}\right)^{2}\right] \\
P_{L}^{p h 12}=P_{n}^{p h 12}\left[c_{1}^{P}+c_{2}^{P}\left(\frac{V^{p h 12}}{\sqrt{3} V_{n}}\right)+c_{3}^{P}\left(\frac{V^{p h 12}}{\sqrt{3} V_{n}}\right)^{2}\right](18) \\
Q_{L}^{p h 12}=Q_{n}^{p h 12}\left[c_{1}^{Q}+c_{2}^{Q}\left(\frac{V^{p h 12}}{\sqrt{3} V_{n}}\right)+c_{3}^{Q}\left(\frac{V^{p h 12}}{\sqrt{3} V_{n}}\right)^{2}\right]
\end{gathered}
$$

Where, $p h 12=a b, b c, c a$.

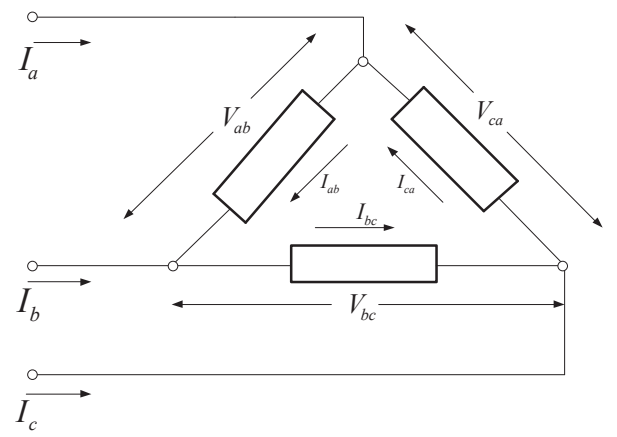

Figure 2. Delta-connected three-phase load

Figure 2 shows a typical $\Delta$-connected three phase load. The voltage magnitudes are line-to-neutral for the state estimation formulation given in Section II. Therefore, in case of delta loads, the equivalent wye powers are calculated at each iteration in order to calculate the active and reactive power at each node. This is illustrated in the following steps.

- Calculate line-to-neutral voltage for delta loads

$$
\left[\begin{array}{c}
V_{i}^{a b} \\
V_{i}^{b c} \\
V_{i}^{c a}
\end{array}\right]=\left[\begin{array}{c}
V_{i}^{a} \angle \delta_{i}^{a}-V_{i}^{b} \angle \delta_{i}^{b} \\
V_{i}^{b} \angle \delta_{i}^{b}-V_{i}^{c} \angle \delta_{i}^{c} \\
V_{i}^{c} \angle \delta_{i}^{c}-V_{i}^{a} \angle \delta_{i}^{a}
\end{array}\right]
$$

- Read the active and reactive power of delta loads

- Calculate the line currents of delta loads

$$
I_{a b}=\left(\frac{P_{a b}+j Q_{a b}}{V_{a b} \angle \delta_{a b}}\right)^{*}
$$

- Calculate the current at each phase

$$
\left[\begin{array}{l}
I_{a} \\
I_{b} \\
I_{c}
\end{array}\right]=\left[\begin{array}{ccc}
1 & 0 & -1 \\
-1 & 1 & 0 \\
0 & -1 & 1
\end{array}\right]\left[\begin{array}{l}
I_{a b} \\
I_{b c} \\
I_{c a}
\end{array}\right]
$$

- Calculate the equivalent line-to-neutral active and reactive powers

$$
\begin{aligned}
V_{a} I_{a}^{*} & =P_{a}+j Q_{a} \\
V_{b} I_{b}^{*} & =P_{b}+j Q_{b} \\
V_{c} I_{c}^{*} & =P_{c}+j Q_{c}
\end{aligned}
$$

For the phases where the loads are non-existent, the active and reactive power values are set to zero for those particular phases.

\section{E. Measurements}

The distribution system normally covers a large geographical area. Hence, it is not possible to place meters at every node and lines. Hence, the redundancy of distribution systems are usually far less than that of transmission systems. However, it is required to make the system observable in order to solve the state estimation. Therefore, the load data taken from historical load data profiles are taken as pseudo measurements and zeroinjection buses are considered as virtual measurements.

\section{CASE STUDY AND DISCUSSIONS}

\section{A. Simulation Results}

A standard IEEE-13 bus distribution system has been used in this paper. The feeders are small yet they show some interesting characteristics. The system represents a typical distribution system with voltage magnitude measurements only at the substation, with more branch current measurements than power flow measurements, and all loads are considered as pseudo measurements.

- $\quad$ Short and relatively high loaded for a $4.16 \mathrm{kV}$ feeder.

- One substation voltage regulator consisting of three single phase units connected in wye.

- $\quad$ Both overhead and underground lines are present with a variety of phasing.

- It has shunt capacitors.

- It has one transformer: grounded wye-grounded wye

- Unbalanced spot and distributed loads are present.

- $\quad$ The loads are of constant power, constant current and constant impedance type and are star and/or delta connected.

Figure 3 shows a typical IEEE 13-bus system. A WLS state estimator is coded in Matlab and tested on the standard IEEE13 bus system and run on a system with Intel Xeon processor @ $3.33 \mathrm{GHz}$ and $12 \mathrm{~GB}$ RAM. The data for the system are given in [12] and [13].

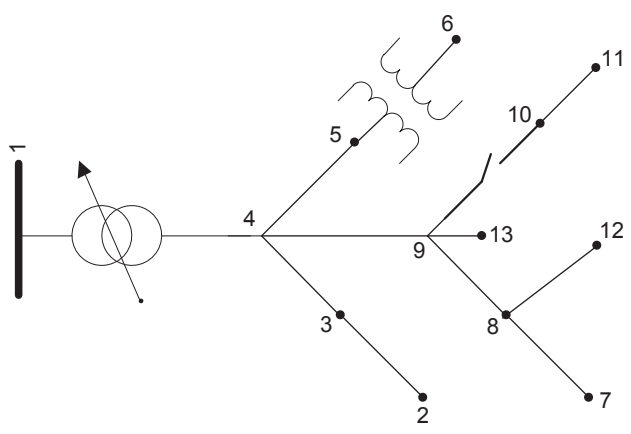

Figure 3. IEEE-13 bus unbalanced distribution system

The overhead lines and underground cables are modelled by modified Carson's equations. The loads are modelled in ZIP model and the three-phase transformer is configured as a grounded Y-Y connection. Measurements have been generated using normal distribution curve with load flow values as true or mean values and standard deviation. Each measurement is taken from the distribution curve randomly and this experiment 
is performed a number of times in a Monte Carlo approach. One such case has been shown here, in the results. It is assumed that the measurement errors are independent and identically distributed. The zero injections are considered as equality constraints. The voltage magnitudes are set to operate within $5 \%$ of the nominal values and the voltage angles within $-30^{\circ}$ to $+30^{\circ}$. The switch between buses 9 and 10 is assumed to be close. Therefore, the equation (14) are also equality constraints in the state estimation formulation. The loads on nodes 2, 9 and 10 are delta-configured loads. The constant impedance loads are on nodes 2 and 10, while the nodes 10 and 7 have constant current loads.

\section{TABLE I. STATE Estimates}

\begin{tabular}{|c|c|c|c|c|c|c|}
\hline & \multicolumn{3}{|c|}{ Angle estimates(in degrees) } & \multicolumn{3}{c|}{ Voltage estimates(in pu) } \\
\hline $\begin{array}{c}\text { Bus } \\
\text { No. }\end{array}$ & $\boldsymbol{p h} \boldsymbol{a}$ & $\boldsymbol{p h} \boldsymbol{b}$ & $\boldsymbol{p h} \boldsymbol{c}$ & $\boldsymbol{p h} \boldsymbol{a}$ & $\boldsymbol{p h} \boldsymbol{b}$ & $\boldsymbol{p h} \boldsymbol{c}$ \\
\hline 1 & 0 & -120 & 120 & 1.0006 & 0.9980 & 1.0030 \\
2 & - & -119.566 & 119.5905 & - & 0.9897 & 1.0068 \\
3 & - & -119.6192 & 119.6037 & - & 0.9899 & 1.0070 \\
4 & -0.2794 & -119.6875 & 119.6423 & 0.9944 & 0.9925 & 1.0076 \\
5 & -0.3005 & -119.7033 & 119.6340 & 0.9920 & 0.9919 & 1.0057 \\
6 & -0.5364 & -119.8673 & 119.4340 & 0.9940 & 0.9923 & 1.0064 \\
7 & - & - & 119.4432 & - & - & 1.0053 \\
8 & -0.6496 & - & 119.4655 & 0.9867 & - & 1.0065 \\
9 & -0.6319 & -119.2681 & 119.4736 & 0.9893 & 0.9930 & 1.0076 \\
10 & -0.6319 & -119.2681 & 119.4736 & 0.9892 & 0.9930 & 1.0076 \\
11 & -0.6519 & -119.2604 & 119.4922 & 0.9891 & 0.9936 & 1.0095 \\
12 & -0.6253 & - & - & 0.9875 & - & - \\
13 & -0.6323 & -119.2676 & 119.4736 & 0.9900 & 0.9950 & 1.0116 \\
\hline
\end{tabular}

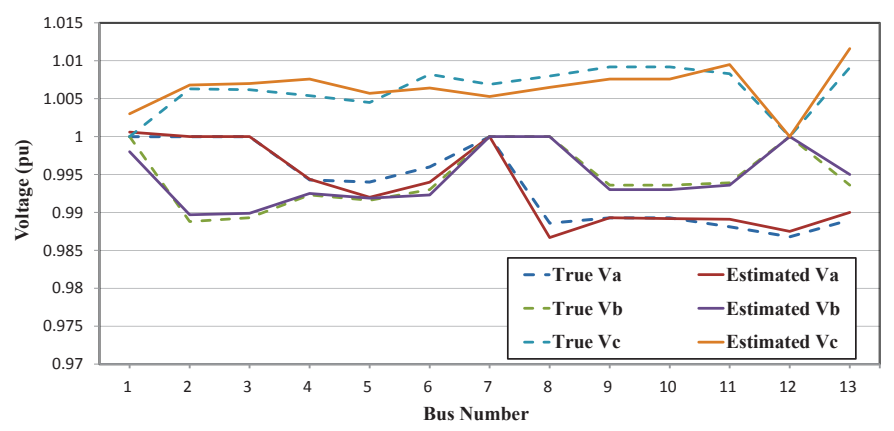

Figure 4. True and estimated voltages for IEEE 13 bus system

Table I present the estimated values of the state variables. The error in real measurement is assumed to be $3 \%$ and the

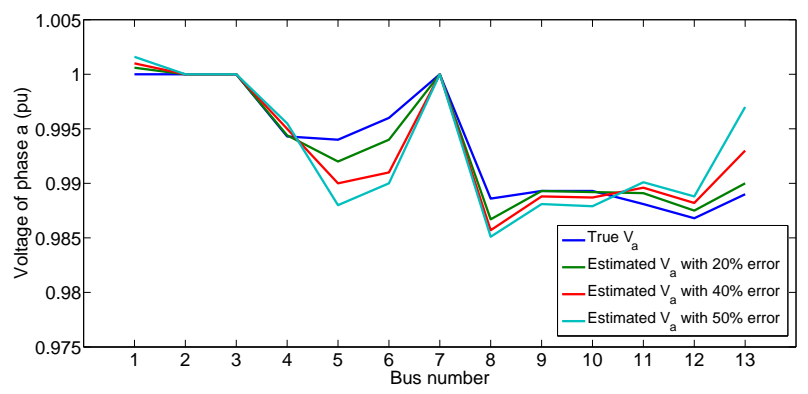

Figure 5. True and estimated voltages for phase a with $20 \% 40 \%$ and $50 \%$ error error in pseudo measurement is assumed to be $20 \%$. Figure 4 shows the true and estimated voltage magnitudes of the three phases for the IEEE-13 bus model. The missing phases have been taken to be of voltage magnitude value equal to 1. However, the state estimation process is performed with $40 \%$ and $50 \%$ error in pseudo measurements as well. Figure 5 shows that for cases when the error in pseudo measurement is large the state estimates are less accurate. The figure shows that when the error is $20 \%$ the estimates for phase $a$ are closest to the true value compared to other cases. In Table I, the missing phases have been represented by dashes. There is a closed switch between bus 9 and 10. Figure 4 and Table I show that the voltage magnitude values and voltage angles remain the same across the closed switch. The obtained results have been found to satisfactory within the allowable tolerance.

\section{Conclusions}

This paper presents a WLS based three-phase state estimation with detailed modelling of the different components of three phase system considering both the star and deltaconfigured loads. The method achieved a reliable solution to the state estimation problem. Simulation results on IEEE 13-bus distribution system showed the effectiveness of the approach and compared with the load flow results. The reliable state estimation results provide the basis for control and monitoring of modern distribution systems.

\section{REFERENCES}

[1] C. Gomez-Quiles, A. Gomez-Exposito, and A. de la Villa Jaen, "State estimation for smart distribution substations," IEEE Transactions on Smart Grid, vol. 3, pp. 986-995, June 2012.

[2] S. S. S. R. Depuru, L. Wang, and V. Devabhaktuni, "Smart meters for power grid: Challenges, issues, advantages and status," Renewable and sustainable energy reviews, vol. 15, no. 6, pp. 2736-2742, 2011.

[3] P. Koponen, L. D. Saco, N. Orchard, T. Vorisek, J. Parsons, C. Rochas, A. Z. Morch, V. Lopes, and M. Togeby, "Definition of smart metering and applications and identification of benefits," Deliverable D3 of the European Smart Metering Alliance ESMA (available at www. esmahome. eu, members area), 2008.

[4] A. Abur and A. Exposito, Power System State Estimation, Theory and Implementation. CRC Press, 2004.

[5] A. Monticelli, State Estimation in Electric Power Systems, A Generalized Approach. Luwer's power Electronics and power Systems Series, 2004.

[6] J. Martinez and J. Mahseredjian, "Load flow calculations in distribution systems with distributed resources. a review," in Power and Energy Society General Meeting, 2011 IEEE, pp. 1-8, July 2011.

[7] R. Singh, B. Pal, and R. Jabr, "Statistical representation of distribution system loads using gaussian mixture model," IEEE Transactions on Power Systems, vol. 25, pp. 29-37, Feb 2010.

[8] C. Lu, J. Teng, and W.-H. Liu, "Distribution system state estimation," IEEE Transactions on Power Systems, vol. 10, pp. 229-240, Feb 1995.

[9] D. Thukaram, J. Jerome, and C. Surapong, "A robust three-phase state estimation algorithm for distribution networks," Electric Power Systems Research, vol. 55, no. 3, pp. 191-200, 2000.

[10] W. H. Kersting, Distribution system modeling and analysis. CRC press, 2012.

[11] P. Xiao, D. Yu, and W. Yan, "A unified three-phase transformer model for distribution load flow calculations," IEEE Transactions on Power Systems, vol. 21, pp. 153-159, Feb 2006.

[12] "Distribution test feeders." http://ewh.ieee.org/soc/pes/dsacom/ testfeeders/index.html.

[13] W. H. Kersting, "Radial distribution test feeders," in Power Engineering Society Winter Meeting, 2001. IEEE, vol. 2, pp. 908-912, IEEE, 2001. 